\title{
Psychologische Behandlungsverfahren bei Kopfschmerz
}

\author{
Entwicklungen im Wandel der Zeit
}

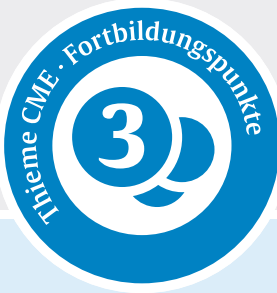

Thomas Dresler ${ }^{1,2,}{ }^{*}$, Timo Klan ${ }^{3 *}$, Eva Liesering-Latta ${ }^{4}$, Johanna Lüpke ${ }^{5}$, Irina Hubalek ${ }^{6}$, Torsten Kraya7, Peter Kropp ${ }^{5}$

1 Klinik für Psychiatrie und Psychotherapie, Universität Tübingen

2 Graduiertenschule \& Forschungsnetzwerk LEAD, Universität Tübingen

3 Psychologisches Institut, Johannes Gutenberg-Universität Mainz

4 Migräne- und Kopfschmerzklinik Königstein

5 Institut für Medizinische Psychologie und Medizinische Soziologie, Universitätsmedizin Rostock

6 Charité - Universitätsmedizin Berlin

7 Klinikum St. Georg, Leipzig

${ }^{*}$ geteilte Erstautorenschaft

\section{ZUSAMMENFASSUNG}

In diesem Beitrag wird beschrieben, welche psychologischen Behandlungsverfahren in der Therapie von Kopfschmerzerkrankungen eingesetzt werden können. Technologische Entwicklungen in den letzten Jahrzehnten habe neue Möglichkeiten eröffnet, deren Potenzial es allerdings noch genauer auszuloten gilt. Die Idee moderner Kopfschmerztherapie ist nicht der isolierte Einsatz einzelner Verfahren, sondern eine möglichst gute, individuell auf den Patienten zugeschnittene Kombination verschiedener psychologischer Behandlungsverfahren mit anderen medikamentösen und nicht medikamentösen Verfahren. Um für jeden Kopfschmerzpatienten eine maßgeschneiderte Therapie zu ermöglichen, sollte ein interdisziplinäres Vorgehen das Ziel sein.

\section{Einleitung}

Psychologische Behandlungsverfahren spielen in der modernen Kopfschmerztherapie eine wichtige Rolle. Dies zeigt sich in den S1-Leitlinien der Deutschen Migräne- und Kopfschmerzgesellschaft (DMKG) zu psychologischen Verfahren in der Behandlung der Migräne [1] sowie der Deutschen Gesellschaft für Neurologie (DGN) und DMKG zur Therapie der Migräneattacke und Prophylaxe der Migräne [2]. So wird in diesen Leitlinien die Anwendung von verhaltenstherapeutischen Verfahren wie Entspannungsverfahren, kognitive Verhaltenstherapie (KVT) oder Biofeedback insbesondere zur Prophylaxe der Migräne empfohlen. Auch in der S1-Leitlinie zur Therapie des episodischen und chronischen Kopfschmerzes vom Spannungstyp [3] werden Entspannungsverfahren und/oder Biofeedback als empfehlenswerte Maßnahmen genannt. In anderen kopfschmerzrelevanten S1-Leitlinien (Clusterkopfschmerz und trigeminoautonome Kopfschmerzen [4], Kopfschmerz bei Übergebrauch von Schmerz- oder Migränemitteln [5]) werden psychologische Behandlungsverfahren hingegen nicht explizit empfohlen, haben im multimodalen Kontext jedoch auch ihre Bedeutung (z. B. Psychoedukation, motivierende psychologische Beratung).

Die Evidenzlage zur Wirksamkeit psychologischer Verfahren bei primären Kopfschmerzen ist nach wie vor nicht zufriedenstellend geklärt. Während mehrere Reviews bzw. Übersichtsarbeiten zur Wirksamkeit verhaltenstherapeutischer Verfahren bei Migräne auf gute Behandlungs- effekte hinweisen [1, 6-8], kommen Sharpe et al. [9] in einem Cochrane-Review zu dem Schluss, dass die Wirksamkeit psychologischer Verfahren zur Migräneprophylaxe nicht ausreichend belegt und die Qualität bisheriger Wirksamkeitsstudien noch ausbaufähig sei. Insbesondere für Follow-up-Erhebungen finden sich in den Cochrane-Reviews bei Erwachsenen [9] und Kindern [10] keine überzeugenden Effekte. Für den Kopfschmerz vom Spannungstyp konnte nachgewiesen werden, dass Biofeedback eine effektive Behandlungsmethode ist [11]. Zu weiteren psychologischen Interventionen bei Kopfschmerz vom Spannungstyp liegen nach unserem Wissen noch keine gesicherten Evidenzen vor. Auch für andere primäre Kopfschmerzerkrankungen (z. B. trigeminoautonome Kopfschmerzen) gibt es kaum randomisiert-kontrollierte Studien, sodass hier noch ein hoher Forschungsbedarf attestiert werden kann. Im Folgenden werden wir psychologische Behandlungsansätze im Wandel der Zeit darstellen, anschließend auf Entwicklungen einzelner Verfahren bzw. Konzepte eingehen.

\section{Ein geschichtlicher Überblick}

Im Rahmen unseres Beitrags werden wir nur die psychologischen Behandlungsansätze und -mechanismen in den Blick nehmen; für pharmakologische und andere medizinische Behandlungsansätze verweisen wir auf diesbezügliche Literatur [12-15]. 


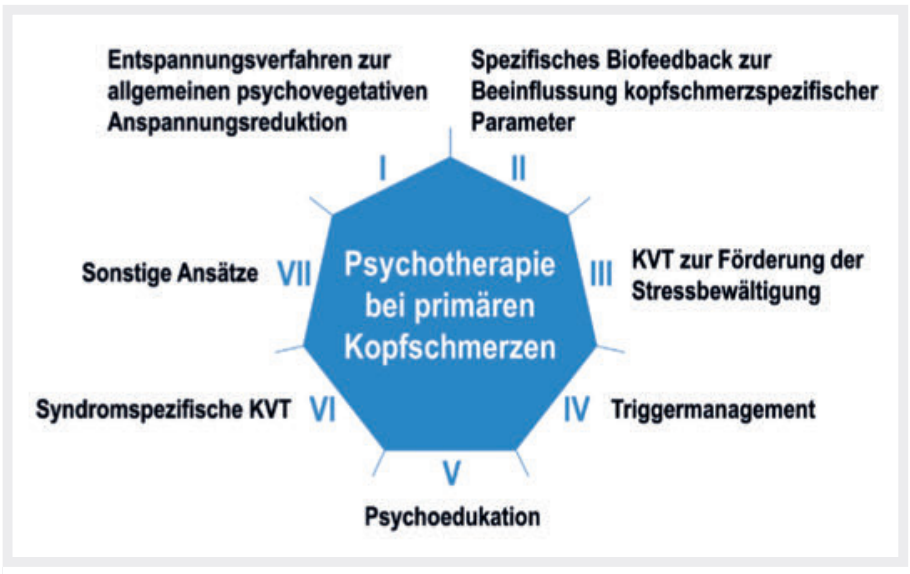

- Abb. 1 Klassifikation von Psychotherapieverfahren bei primären Kopfschmerzen.

Erste überlieferte Berichte von Kopfschmerzbehandlungen datieren zurück bis 2500 v. Chr. (Ebers Papyrus) [14]. Als Ursache von Kopfschmerzen (und anderer Erkrankungen) wurden übersinnliche Phänomene - wie der Einfluss böser Geister oder Dämonen - herangezogen. In der Behandlung wurden dementsprechend Beschwörung und Beten eingesetzt. Es finden sich also bereits in frühen Epochen Vorformen der Psychotherapie i. S. einer primitiven Heilkunst (z. B. suggestive Anwendungen, Traumareproduktionen, Rituale); als Psychotherapeut fungiert ein „Heiler“ [16]. Hippocrates (ungefähr 460-370 v. Chr.) empfahl gegen Kopfschmerzen die Modifikation von kopfschmerzauslösenden Aktivitäten [14]. Dies kann aus heutiger Sicht als ein erster Einsatz verhaltenstherapeutischer Methoden bei Kopfschmerzen angesehen werden. Ab dem 18. Jahrhundert entwickelte sich - beginnend mit Franz Anton Mesmer (1734-1815) - eine Ausdifferenzierung hypnotischer Ansätze, welche später auch Sigmund Freud (1856-1939) beeinflussten. Dieser begründete Ende des 19. Jahrhunderts die Psychoanalyse, welche als Ausgangspunkt der modernen Psychotherapie verstanden werden kann [16]. Nachfolgend kam es zu der Herausbildung verschiedener psychotherapeutischer Strömungen. Begünstigt wurde dies auch durch die zunehmende Etablierung psychologischer Institute und wissenschaftlich-experimenteller Ansätze.

In der Mitte des 20. Jahrhunderts entwickelte sich die Verhaltenstherapie (VT). Einerseits, weil eine rasche Entwicklung und starke Produktivität in der Grundlagenforschung zu lerntheoretischen Erklärungen stattfand (Epoche des Behaviorismus); andererseits, weil zunehmend kritische Stimmen zur Effektivität psychoanalytischer Methoden laut wurden. Der Begriff Verhaltenstherapie wurde 1958 erstmals von Arnold Lazarus (1932-2013) in einer wissenschaftlichen Fachzeitschrift verwendet. Die aufsehenerregenden Erfolge der Verhaltenstherapie, insbesondere im Bereich der Behandlung von Ängsten, führten zu einer raschen Weiterentwicklung ihrer Methoden [17]. Ursprüng- lich beinhaltete die Verhaltenstherapie die Anwendung vorwiegend basaler Lernmechanismen und -prinzipien, die sowohl bei Tier und Mensch untersuchbar sind und sich primär auf beobachtbares Verhalten beziehen; höhere kognitive Prozesse wurden hingegen kaum betrachtet („erste Welle“ der VT). Im Rahmen der Kognitiven Wende in den 1960er-Jahren wurden kognitive Variablen (z. B. Gedanken, Erwartungen, bestimmte Denkmuster) als zunehmend bedeutsamer angesehen und in die Verhaltenstherapie aufgenommen. Auch deswegen, weil rein verhaltenstherapeutische Therapien ohne den Einbezug kognitiver Variablen weniger Erfolg erzielten. Es etablierte sich hierdurch die KVT („zweite Welle“ der VT). Nachfolgend gab es immer wieder Weiterentwicklungen von verhaltenstherapeutischen Verfahren, die unter dem Begriff der „dritten Welle“ der VT subsumiert werden [17].

\section{Hin zu einer Kopfschmerzpsycho- therapie}

Ein wichtiger Schritt hin zu einer spezifischeren Schmerztherapie war die Entwicklung eines biopsychosozialen Modells, welches das enge Zusammenspiel zwischen körperlichen, psychischen und sozialen Faktoren bei der Entstehung und Aufrechterhaltung von Schmerzerkrankungen berücksichtigt. Mit diesem konnten sehr unterschiedliche Zugänge zum Symptom (Kopf-)Schmerz und damit zu seiner Behandlung beschrieben werden [18, 19]. Insbesondere bei der Aufrechterhaltung und Chronifizierung von Schmerzen spielen Lernvorgänge eine bedeutsame Rolle. Deshalb sind die therapeutischen Verfahren auf ein Verlernen bzw. eine Veränderung der schmerzauslösenden, -verstärkenden oder -aufrechterhaltenden Bedingungen im Verhalten und Erleben der Patienten gerichtet [20]. Zusätzlich spielen Aufmerksamkeitsprozesse eine Rolle. So kann die Umlenkung der Aufmerksamkeit auf nicht schmerzassoziierte Erlebnisinhalte im Sinne einer Ablenkung zu einer höheren Schmerztoleranz und verbesserten Schmerzbewältigung beitragen, was in Schmerzbewältigungstrainings und teilweise auch Entspannungstechniken angewandt wird. Psychophysiologische Forschung [21] erbrachte bei Migräne Hinweise auf erhöhte kortikale Aufmerksamkeitszustände und reduzierte Habituationsneigungen im schmerzfreien Intervall, die sich als Reizverarbeitungsstörung interpretieren lassen. Als Konsequenz daraus lassen sich Behandlungsverfahren entwickeln, die mit Entspannung assoziiert sind.

Im Hinblick auf die Psychotherapie bei Kopfschmerzen können gegenwärtig folgende Kategorien unterschieden werden ( $\triangleright$ Abb. 1): Entspannungsverfahren zur allgemeinen psychovegetativen Anspannungsreduktion, (z. B. progressive Muskelrelaxation, meditative Verfahren, Temperatur-Biofeedback), spezifisches Biofeedback zur Beeinflussung kopfschmerzspezifischer Parameter (z. B. Neurofeedback/CNV-Biofeedback), KVT zur Förderung der Stressbewältigung, Triggermanagement, Psy- 


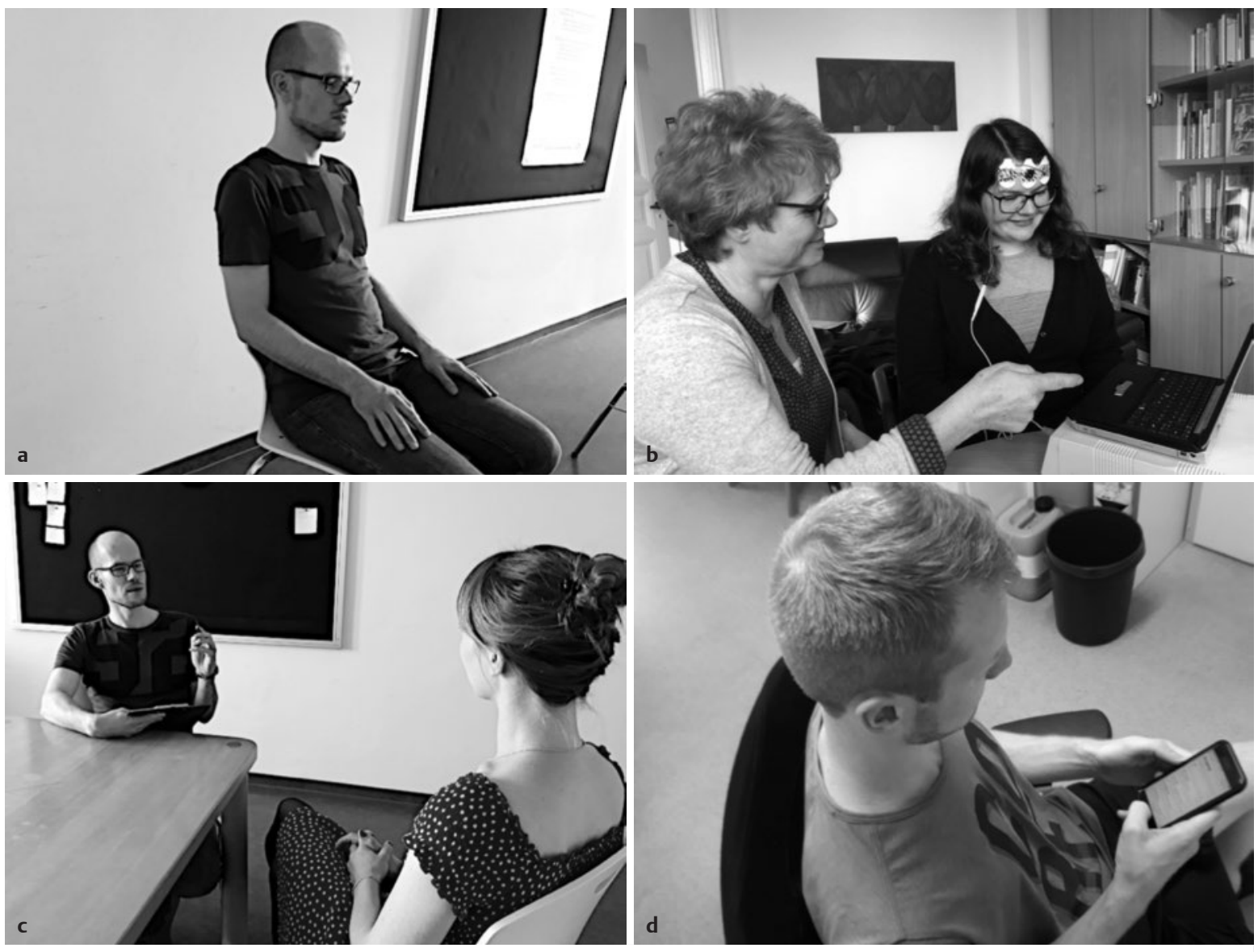

- Abb.2 Anwendungsbeispiele psychologischer Methoden in der Behandlung von Kopfschmerz: a - angeleitete Entspannungsverfahren, b - elektromyografisches Biofeedback, c - Einzelsitzung KVT, d - neue Technologien (z. B. Migräne-App). Quelle: Beatrix Barth, Peter Kropp

choedukation, syndromspezifische KVT (z. B. Verfahren zur Bewältigung von Attackenangst, Attackenmanagement, störungsspezifische Lebensstilempfehlungen wie Ausdauersport), sonstige Ansätze wie z. B. psychodynamische Verfahren, Akzeptanz- und Commitmenttherapie (ACT). Eine Klassifikation psychologischer Verfahren zur Behandlung von Kopfschmerzen wurde bislang von verschieden Autoren vorgenommen [1, 22-24]. Letztendlich stellen die genannten Kategorien keine klar abgrenzbaren Bereiche dar. So sind psychoedukative Elemente meistens auch anderen Ansätzen immanent (z. B. Psychoedukation vor Durchführung von Biofeedback). Auch weitere Kategorien beinhalten Elemente aus anderen Bereichen (z. B. umfasst das Triggermanagement KVT zur Stressbewältigung). Während für einige Verfahren (Entspannung, KVT zur Stressbewältigung, Biofeedback) bei bestimmten Kopfschmerzerkrankungen (hier: Migräne) die Evidenzlage durchaus gut ist [1], fehlen für andere Verfahren respektive andere Kopfschmerzerkrankungen noch jegliche Evidenznachweise. Auch wenn die syndromspezifischen KVT-Verfahren augenscheinlich sinnvoll und oft indiziert sein dürften (z. B. Verbesserung des Attackenmanagements), gibt es kaum Nachweise für deren Effektivität. Empirische Belege zur Wirksamkeit einzelner Verfahren sind sicherlich schwierig zu generieren, da viele Elemente in der Versorgungspraxis und in Therapieprogrammen oft im Verbund (z. B. Entspannungsverfahren in Kombination mit KVT zur Stressbewältigung und syndromspezifischen Verfahren) eingesetzt werden. Nachfolgend werden wir - dem geschichtlichen Pfad folgend - wesentliche Komponenten psychologischer Kopfschmerzbehandlung vorstellen ( $\triangleright$ Abb. 2).

\section{Entspannungsverfahren}

Entspannungsverfahren haben das Ziel, das allgemeine Aktivierungsniveau zu reduzieren. Neben einer allgemeinen entspannenden Wirkung wird hierbei eine zentrale Dämpfung der Informationsverarbeitung angestrebt [25]. Bildgebende Studien zeigten, dass Entspannungsstrategien bei akuten Schmerzen zu reduzierten Schmerzangaben, aber auch zu einer erhöhten Aktivierung im periaquaeduktalen Grau führen - einer Region, die in der endogenen kortikalen Schmerzkontrolle involviert ist [26]. Entspannung kann Angstzustände reduzieren, was wiederum die Schmerztoleranz erhöht und somit das Schmerzerleben reduziert. Häufig wird Entspannungsverfahren bei Schmerzen eine präventive Funktion (i. S. einer Prophylaxe) zuge- 
sprochen; zudem berichten Patienten über abortive Eigenschaften bei akuten Schmerzzuständen [20].

Entspannungsverfahren benötigen einen vergleichsweise geringen Therapeutenaufwand; sie können in Gruppen vermittelt und vom Patienten selbstständig zu Hause weiter geübt werden. Jeder Patient sollte das für ihn geeignete Entspannungsverfahren finden, denn die subjektive Erwartung hinsichtlich einer Verbesserung trägt maßgeblich zum Erfolg bei [27].

\section{Autogenes Training}

Das Autogene Training (AT) wurde in den 1920er-Jahren von Johannes Heinrich Schultz (1884-1970) entwickelt und ist eine der verbreitetsten Methoden zur Entspannungsinduktion. Dabei ist das AT eine Form der vereinfachten und standardisierten Selbsthypnose, die auf den Mechanismen der klassischeren Konditionierung basiert. Suggestive Formelsätze werden als Stimuli mit der Reaktion der Entspannung verbunden, sodass nach mehrmaligem Üben bereits ein Entspannungszustand eintritt, wenn die Formelsätze gesagt bzw. gedacht werden. Das Ziel der Arbeit mit diesen suggestiven Formelsätzen ist, über mentale Instruktionen den Körper zu beeinflussen und so eine konzentrative Selbstentspannung herbeizuführen.

In der Grundstufe des AT [28] werden 7 Grundübungen (Ruhe, Schwere, Wärme, Atem, Herz, Sonnengeflecht/ Bauch, Kopf/Stirn) benutzt, die aufeinander aufbauen und deshalb nacheinander erarbeitet und eingeübt werden sollen. Zu jeder Grundübung gibt es Autosuggestionen (z. B. zur Ruhe: „Ich bin vollkommen ruhig und entspannt“ und zum Herz: „Mein Herz schlägt ruhig und gleichmäßig“), die jeweils 6-mal wiederholt werden. Jede Grundübung sollte mindestens eine Woche lang, 2- bis 3-mal täglich, durchgeführt werden, bis die nachfolgende Grundübung zusätzlich praktiziert werden kann [29].

Die Wirksamkeit der Grundstufe des AT wurde durch metaanalytisch gewonnene Befunde [30] für eine Großzahl von psychischen und somatischen Krankheitsbildern belegt. In einem kürzlich erschienen Review [31] konnte gezeigt werden, dass AT in 5 von 6 Studien signifikant Kopfschmerzen bei Erwachsenen reduziert. Dabei wurden in den jeweiligen Studien unterschiedliche Formen von Kopfschmerzen und die Anwendungsdauer des AT, auch in Kombination mit Biofeedback, untersucht. Besonders hinsichtlich der optimalen Länge des AT für die jeweiligen Kopfschmerztypen stehen noch weitere Untersuchungen aus. Zwei koreanische Studien fanden, dass das AT in Kombination mit Biofeedback bei Spannungskopfschmerz [32] und Migräne [33] wirksam ist. Auch ohne zusätzliches Biofeedback konnte eine signifikante Reduktion von Kopfschmerzen gefunden werden [34]. Die Durchführung von AT in der Migräneattacke ist sorgfältig abzuwägen, da die kardiovaskuläre Entspannungsreaktion mit einer peripheren Gefäßerweiterung einhergehen kann.

\section{Progressive Muskelrelaxation}

Die progressive Muskelrelaxation (PMR) nach Edmund Jacobson (1888-1983), heute überwiegend nach den Empfehlungen von Bernstein und Borkovec [35] angewandt, wird in der Schmerztherapie am häufigsten als Entspannungsverfahren eingesetzt [36]. Hierbei werden - in der Langversion (Dauer: ca. 20 Minuten) - insgesamt 16 Muskelgruppen des Körpers identifiziert und für wenige Sekunden angespannt, es folgt eine 30 - bis 40-sekündige Entspannungsphase. Der Patient konzentriert sich auf die wahrgenommenen körperlichen Unterschiede zwischen Anspannung und Entspannung. Auf diese Weise kann sich eine allgemeine psychovegetative Entspannungsreaktion einstellen. Es existieren verschiedenen Kurzversionen, bei denen mehrere Muskelgruppen zusammengefasst werden (Dauer: max. 5 Minuten).

Zusätzlich gibt es eine „Vergegenwärtigungsübung“, bei der sich der Patient vor dem Entspannungsintervall lediglich die vorhandene Muskelspannung bewusst macht, allerdings die Muskeln nicht mehr anspannt. Dies kann dann - bei geübten Personen - unmittelbar und schnell eingesetzt werden, eine Entspannung kann binnen Sekunden erfolgen. Die PMR wird seit vielen Jahrzehnten in der Migränetherapie eingesetzt. Auf der Basis vieler Untersuchungen wird die Anwendung auch in den entsprechenden Leitlinien zur Migränebehandlung empfohlen [1, 2]. Ein Vorteil der PMR ist, dass sie nach dem Erlernen ohne Therapeuten und technische Hilfsmittel beinahe überall und sofort eingesetzt werden können. Im Vergleich zum AT eignet sich die PMR mehr für Patienten mit erhöhter Anspannung und Nervosität [37].

\section{Meditation}

Während Meditation in der Vergangenheit vornehmlich in einem primär-präventiven Anwendungskontext eingesetzt wurde, werden mit der „dritten Welle“ der VT Meditationselementen eine zunehmend größere Bedeutung für den therapeutischen Kontext zugeschrieben. Mit neueren Therapieverfahren, wie der dialektisch-behavioralen Therapie (DBT), der ACT sowie der achtsamkeitsbasierten kognitiven Therapie (engl. Mindfulness Based Cognitive Therapy; $\mathrm{MBCT}$ ) werden erfolgreich meditative Praktiken mit buddhistischer Prägung in die Behandlung eines breiten Spektrums von Erkrankungen integriert. Meditationstechniken finden sich auch in Programmen zur individuellen Stressbewältigung wieder, z. B. im Stressreduktionstraining mit Yogaelementen für Erwachsene [38]. Zusätzlich ist in den vergangenen Jahren ein großes gesellschaftliches Interesse an buddhistischen Meditationstechniken zu beobachten, das sich exemplarisch in dem rapide zunehmendem Angebot an vielfältigen Meditations-Apps abbildet. Für die Behandlung von Kopfschmerzpatienten wird das Potenzial der Meditation jedoch noch unzureichend genutzt, obwohl sich die Meditation bereits in der Therapie von Schmerzpatienten als wirksam gezeigt hat [39]. 
Meditationstechniken zielen auf einen Zustand der Vorstellungslosigkeit, der geschärften Innenschau (Introspektion) und/oder der höchsten Konzentration. Damit gehen sie über das Ziel der zuvor dargestellten Entspannungsverfahren hinaus. Meditation kann als eine Exposition mit dem eigenen Selbst verstanden werden, indem sie die Introspektionsfähigkeit fördert. Ziel ist es über die Meditation die Beobachtung für sich selbst zu schärfen, offener für die Gegenwart zu werden und mehr im Jetzt zu sein. Gerade für Kopfschmerzpatienten ist dies essenziell, um Hinweise auf Kopfschmerzattacken frühzeitig wahrzunehmen und entsprechend zu handeln. Es gibt unterschiedliche Formen, wie die Meditation im Sitzen (mit Atembeobachtung [Vipassana], Mantra [Silben], Klang oder Objekten [z. B. Kerze]) sowie die Meditation in Bewegung (z. B. Gehmeditation). Meditationsverfahren bestehen oft aus Körperübungen (z. B. Lotussitz, Atemtechniken) und aus Vorstellungsübungen. Dabei werden zur Selbstinstruktion Mantras, sich monoton wiederholende Wortfolgen geformt, die einen Rhythmus bezeichnen, der die Entspannung fördert.

Der mit Meditation erreichte Entspannungszustand ist physiologisch mit AT und PMR vergleichbar [40]. Auch bei Mediation erhöht häufiges und regelmäßiges Üben die Wirksamkeit und führt dazu, Belastungssituationen frühzeitiger zu erkennen und ihnen gelassener entgegenzutreten. Gerade durch die unterschiedlichen Formen der Meditation und durch die Unterstützung von neu entwickelten Meditations-Apps lässt sich Meditation individuell angepasst in den persönlichen Alltag integrieren.

\section{Biofeedback}

Biofeedback könnte aufgrund seiner Entspannungskomponente auch im vorherigen Kapitel beschrieben werden; hier möchten wir aber auf die spezifische Modulation von Kopfschmerzparametern durch Biofeedback fokussieren.

Durch Biofeedback werden körperliche Prozesse („Bio“) gemessen und dem Patienten kontinuierlich über ein gut wahrnehmbares, meist visuelles oder akustisches Signal zurückgemeldet („Feedback“) [41]. Biofeedback ist deswegen ein objektives Verfahren zur Messung, Verstärkung und Rückmeldung physiologischer Signale. Die Idee ist, dass der Patient diese rückgemeldeten Signale anwendet, um dadurch Kontrolle über eine bestimmte Zielgröße (z. B. Muskelspannung oder Erregungsniveau) zu erhalten. Neben diesen eher unspezifischen Parametern können auch kopfschmerzspezifische Parameter verwendet werden, die bei Migräne verändert sind. Exemplarisch zu nennen sind die contingent negative variation (CNV), ein langsames aufmerksamkeitskorreliertes Potenzial sowie die Gefäßweite der oberflächlichen Schläfenarterie [21, 41]. Diese Parameter sind in die gewünschte Richtung (z. B. weniger Anspannung, Amplitudenreduktion, Gefäßverengung) zu verändern; verschiedene Strategien können angewendet werden (z. B. Herausfinden einer passenden Kognition). Diese Strategien werden durch Versuch und Irrtum erworben; eine Vorgabe wird als eher ungünstig beurteilt [42].

Das Biofeedbacktraining ist ein wesentlicher und wirkungsvoller Baustein verhaltenstherapeutischer Schmerzbehandlung. Dabei geht es um eine Form des Lernens mit verbesserter und gesteigerter Autoregulation. Ungeklärt ist jedoch, ob es sich dabei um eine apparativ unterstützte Entspannungsmethode oder einem Körperwahrnehmungstraining und einer dadurch induzierten kognitiven Umstrukturierung handelt (wie bei der KVT). Bei der Behandlung von Migräneattacken ist die Evidenz von Biofeedback sehr hoch; die Wirksamkeit in der Prophylaxe von Migräneanfällen ist vergleichbar mit einer medikamentösen Behandlung [2]. Als Wirkmechanismen von Biofeedback werden die Kontrolle über physiologische Funktionen als spezifische Anwendung und die Überzeugung einer Symptomkontrolle als unspezifische Anwendung eingesetzt; letztere scheint dabei effektiver zu sein $[23,43]$.

\section{Kognitive Verhaltenstherapie}

Als Begründer der modernen KVT können Albert Ellis (1913-2007) (Rational-Emotive Therapie, 1950er Jahre), Aaron T. Beck ( ${ }^{*} 1921$ ) (Kognitive Therapie, 1950er- bis 1960er-Jahre) und Donald Meichenbaum ( $\left.{ }^{*} 1940\right)$ (Stressimpfungstraining, 1970er-Jahre) genannt werden [44]. Einen Meilenstein für die KVT mit Schmerzpatienten stellt das „Marburger Schmerzbewältigungsprogramm “ [45] dar. Darin wurden erstmals für den deutschen Sprachraum in gut strukturierter und umfassender Form verhaltenstherapeutische Interventionen bei Kopf- und Rückenschmerzen beschrieben (z. B. Psychoedukation, Entspannungsverfahren, Aufmerksamkeitslenkung, Verhaltensanalyse und -modifikation von Schmerzauslösern). Nachfolgende KVT-Programme für Kopfschmerzpatienten stellen eine Ausdifferenzierung und Spezifizierung von KVT-Methoden dar. So wurden inzwischen auch spezifische Therapieprogramme für Kinder und Jugendliche mit Kopfschmerzen entwickelt [46, 47], wobei DreKiP („Ein ambulantes Therapieprogramm für Kinder und Jugendliche mit Kopfschmerz") neben Psychoedukation, Entspannungsverfahren und Stressbewältigung auch erlebnispädagogische Elemente („Klettern und Selbstwirksamkeit“) beinhaltet.

Von hoher Relevanz ist die Frage, welche Faktoren Kopfschmerzen respektive -attacken auslösen. In einer Metaanalyse konnte gezeigt werden, dass am häufigsten Stress oder mit Schlaf assoziierte Faktoren als Auslöser („Trigger“) genannt werden [48]. Weitere häufig berichtete Trigger sind emotionale Belastungen, Wetterwechsel, visuelle Reize (z. B. Bildschirmarbeit), hormonelle Schwankungen, bestimmte Nahrungsmittel sowie körperliche Aktivität/ Anstrengung. Ausgehend von der Annahme, dass das ausschließliche Vermeiden von Triggern kontraproduktiv ist 
und zur Chronifizierung einer Kopfschmerzerkrankung beitragen kann, entwickelte Paul Martin das spezifisch an Kopfschmerzpatienten adressierte Triggermanagement („Learning to Cope with Triggers, LCT“ $[49,50]$ ). Theoretische Grundlage ist Martins Trigger Avoidance Model of Headaches (TAMH, [49]). Dieses beschreibt, wie übermäßige Triggervermeidung zu einer Sensitivierung hinsichtlich dieser Trigger führt und somit die Zunahme von Kopfschmerzaktivität erklären kann. Experimentelle Befunde konnten diese Annahme bestätigen [51-53]. In einer randomisiert-kontrollierten Studie konnte die Wirksamkeit des Triggermanagements und dessen Überlegenheit gegenüber einer ausschließlichen Vermeidungsstrategie dokumentiert werden [54]. Das Triggermanagement zielt auf eine Flexibilisierung im Umgang mit potenziellen Triggern ab. Mit dem Akronym EASE werden 4 Methoden unterschieden [55]:

- Experiment (i. S. eines Verhaltensexperiments hinsichtlich des tatsächlichen Auslösepotenzials),

- Vermeidung (Avoid),

- Stressbewältigung und

- Exposition (i. S. einer graduierten Konfrontation mit dem Trigger).

Mit der Zielsetzung, ein noch spezifischeres KVT-Behandlungsprogramm zu konzipieren, wurde das Migränemanagement (MIMA) entwickelt [24, 56]. Dieses, sich an erwachsene Patienten richtende Therapieprogramm, umfasst 7 modulartig aufgebaute Sitzungen und integriert verschieden KVT- sowie Entspannungsverfahren. Dabei wird zum Teil sehr spezifisch auf migränerelevante Aspekte eingegangen (z. B. Vermittlung eines Entstehungsmodells der Migräne, Bewältigung von Attackenangst, Durchführung von migränespezifischen Basismaßnahmen, Attackenbewältigung, Triggermanagement). In einer Pilotstudie konnte eine sehr gute Durchführbarkeit und Patientenakzeptanz hierfür nachgewiesen werden [24]. Zur Beurteilung der Wirksamkeit des MIMA läuft gegenwärtig eine randomisiert-kontrollierte Studie (DRKS-ID: DRKS00011111).

Insgesamt lässt sich bei KVT-Verfahren ein Trend zu zunehmend spezifischeren Behandlungsprogrammen beobachten. Ein Vorteil dieser Entwicklung ist die Möglichkeit, im Kontext der Kopfschmerztherapie auf die Bedürfnisse von Subgruppen noch adäquater eingehen zu können (z. B. Migränepatienten vs. Clusterkopfschmerzpatienten oder Migränepatienten mit hoher vs. geringer Triggervermeidung).

\section{Multimodale Behandlung}

Multimodalen interdisziplinären Behandlungen liegt das biopsychosoziale Krankheitsverständnis zugrunde [19]. Bereits vor über 20 Jahren wurden multimodale Therapieansätze, v. a. im Bereich chronischer Rückenschmerzen, entwickelt und evaluiert [57]. Nach der Ad-hoc-Kom- mission „Interdisziplinäre Multimodale Schmerztherapie (MMST) “ der Deutschen Schmerzgesellschaft (DGSS) wird als MMST „die gleichzeitige, inhaltlich, zeitlich und in der Vorgehensweise aufeinander abgestimmte umfassende Behandlung von Patienten mit chronifizierten Schmerzsyndromen bezeichnet, in die verschiedene somatische, körperlich übende, psychologisch übende und psychotherapeutische Verfahren nach vorgegebenem Behandlungsplan mit identischem, unter den Therapeuten abgesprochenem Therapieziel eingebunden sind. " [58, S. 112]. Im Operationen- und Prozedurenschlüssel (OPS) medizinischer Leistungen wurde die MMST unter dem Komplexkode 8-918 aufgenommen, welcher u. a. die Integration mindestens einer psychotherapeutischen Fachrichtung, eines multidisziplinären Aufnahmeverfahrens sowie regelmäßiger interdisziplinärer Teambesprechungen fordert. Ziel der MMST ist neben der Symptomlinderung und Förderung eines biopsychosozialen Krankheitsverständnisses die Verbesserung der objektiven und subjektiven Funktionsfähigkeit („functional restauration“).

Die Effektivität interdisziplinärer multimodaler Behandlungsansätze ist insbesondere für chronische Rückenschmerzen und das Fibromyalgiesyndrom vielfach überprüft und belegt worden [59]. Auch in der Behandlung chronischer bzw. komplexer Kopfschmerzen haben sich multimodale Ansätze als wirksam erwiesen, wobei sich die Studienlage vorwiegend auf Migräne, Spannungskopfschmerz und Kopfschmerz durch Medikamentenübergebrauch bezieht. Aussagen über die optimale inhaltliche und organisatorische Ausgestaltung (z. B. notwendige Anzahl der Therapieeinheiten) sind noch nicht möglich [60]. Interdisziplinäre multimodale Kopfschmerzbehandlungen ( $\triangleright$ Abb. 3) erfolgen aktuell ambulant im Rahmen von Programmen der „Besonderen Versorgung“ (früher Integrierte Versorgung) sowie im tagesklinischen oder stationären Behandlungssetting. Für eine ausführliche Darstellung der interdisziplinären Behandlungsbausteine in der multimodalen Therapie chronischer Kopfschmerzen wird auf den praxisnahen Therapieleitfaden von Fritsche und Gaul [61] verwiesen.

Edukation i. S. einer Vermittlung von Wissen über spezifische Kopfschmerzsyndrome, biopsychosoziale Zusammenhänge und medikamentöse sowie nicht medikamentöse Behandlungsansätze spielt eine Schlüsselrolle und zielt v. a. auf eine Steigerung der Selbstwirksamkeitserwartung ab. Idealerweise sollten alle an der MMST beteiligten Berufsgruppen (z. B. Ärzte, Psychotherapeuten, Physiotherapeuten, Pflegekräfte) involviert werden. In einer Metaanalyse [63] konnte gezeigt werden, dass allein mit Psychoedukation bereits Therapieeffekte bei Migränepatienten erreichbar sind (z. B. Reduktion der Kopfschmerzhäufigkeit und kopfschmerzbedingten Funktionsbeeinträchtigung). 
KVT-Methoden lassen sich sehr gut in multimodale Programme integrieren. Meist findet die Behandlung als Therapie in geschlossenen Kleingruppen von Kopfschmerzpatienten statt, mit mehreren strukturierten Sitzungen. Die Patienten werden angeleitet, ungünstige kopfschmerzfördernde Lebensstilfaktoren und Einstellungen zu identifizieren und zu verändern. Weitere Inhalte können sich z. B. auf die Schmerz- und Stressbewältigung, das Triggermanagement und die Stärkung der Krankheitsakzeptanz beziehen. In zusätzlichen psychotherapeutischen Einzelsitzungen können Inhalte vertieft bzw. individualisiert werden und bei Bedarf weiterführende ambulante psychotherapeutische Behandlungen eingeleitet werden.

Perspektivisch werden mehr Studien zu optimalem Inhalt, Umfang und Setting multimodaler Therapieansätze in Abhängigkeit der vorliegenden Kopfschmerzsymptomatik benötigt. Ein interessantes Behandlungsdesign wurde in einer prospektiven Studie im Rahmen der Integrierten Versorgung gewählt [64]. Hierbei wurden Kopfschmerzpatienten (Migräne, Spannungskopfschmerz, Migräne und Spannungskopfschmerz, Kopfschmerz durch Medikamentenübergebrauch) in Abhängigkeit der Anzahl ihrer Kopfschmerztage/Chronifizierung, dem Medikamenten(über) gebrauch und der ggf. begleitenden psychiatrischen Komorbidität einem von 3, modular aufgebauten Behandlungsarmen zugeteilt. Es konnten jeweils signifikante Verbesserungen hinsichtlich der Kopfschmerzhäufigkeit, Reduktion der Akutmedikation und krankheitsbedingten Beeinträchtigung erzielt werden. Nicht zuletzt stellt die Verbesserung von Versorgungsstrukturen wie die Ausweitung niederschwelliger ambulanter interdisziplinärer Behandlungen eine wichtige Aufgabe dar, um einer Chronifizierung und fortschreitenden kopfschmerzbedingten funktionalen und affektiven Beeinträchtigung möglichst frühzeitig entgegenzuwirken.

\section{Diagnostische Aspekte}

Bei der psychologischen Kopfschmerzdiagnostik können folgenden Bereiche unterschieden werden:

- Schmerzsymptomatik (Kopfschmerzaktivität),

- kopfschmerzassoziierte Beeinträchtigung,

- psychische Belastung,

- Krankheitsverarbeitung und

- Einflussfaktoren auf die Kopfschmerzaktivität.

Seit den 1990er-Jahren wurden und werden diesbezüglich zunehmend spezifischere Verfahren entwickelt: z. B. MIDAS (Migraine Disability Assessment Scale [106]), IBK (Inventar zur Beeinträchtigung durch Kopfschmerzen [105]), HIT-6 ${ }^{\text {TM }}$ (Headache Impact Test $\left.[103,104]\right)$ zur Beurteilung der kopfschmerzbedingten Beeinträchtigung sowie HTSAQ-G (Headache Triggers Sensitivity and Avoidance Questionnaire [65]), HMSE-G (Headache Management Self-Efficacy Scale [66]). Aktuell befindet sich ein Testverfahren zur Erfassung von Belastung, Beeinträchti-

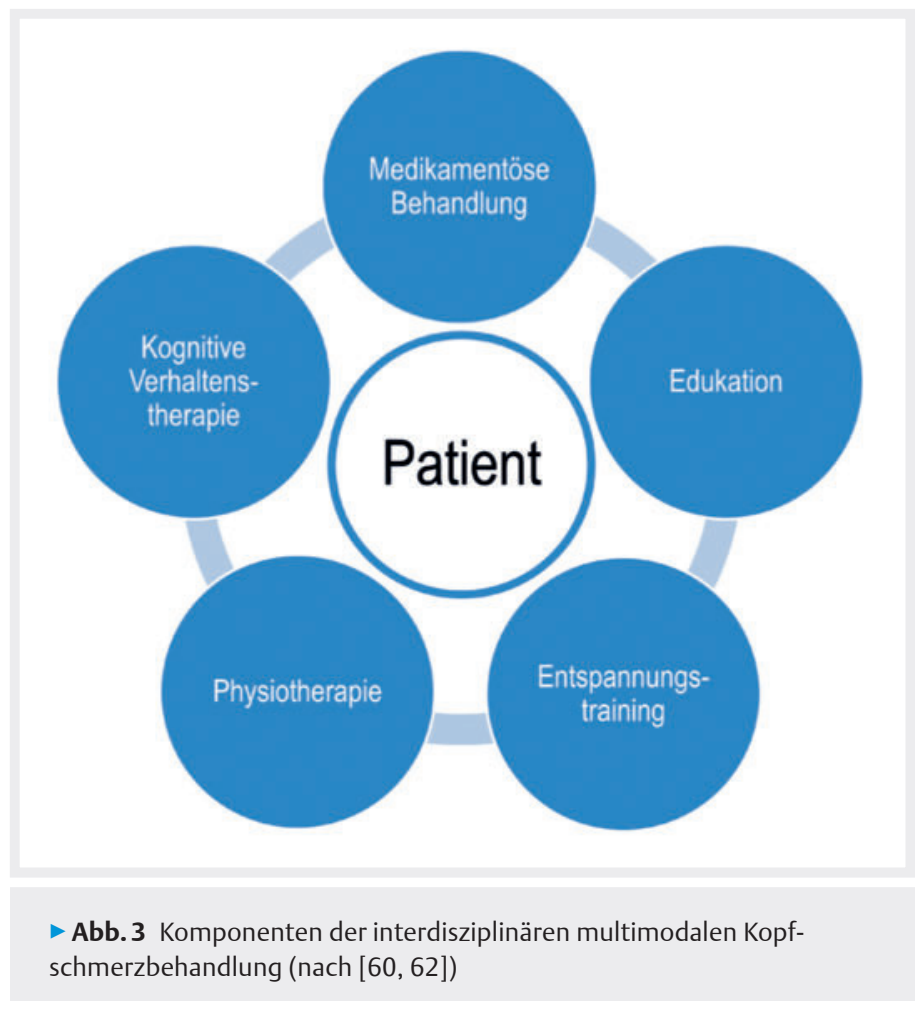

gung und Bewältigung bei Clusterkopfschmerz in der Vorbereitung [67]. Möglicherweise könnte in einigen Jahren bei Migränepatienten die Vorhersage der Kopfschmerzaktivität anhand eines Monitorings der täglichen Stressbelastung eine realistische Option zur Verbesserung der Krankheitsbewältigung sein [68].

\section{Aktuelle technologische Entwicklungen}

Eine schnelle Entwicklung sowie große Verfügbarkeit der Onlinetechnologien und digitalen Medien in der Medizin [69] begünstigten auch in der Kopfschmerztherapie die Herausbildung neuer Formate [70, 71]. Diese werden häufig als eHealth (electronic health) bzw. - bezogen auf mobile Endgeräte - als mHealth (mobile health) bezeichnet und können insbesondere genutzt werden, um bewährte nicht medikamentöse Konzepte zu vermitteln. Den Gang zum Therapeuten können sie jedoch nicht ersetzen [72]. Sie sollten als unterstützende Möglichkeiten im diagnostischen und therapeutischen Prozess angesehen werden, mit dem Potenzial einer verbesserten Patientenversorgung (z. B. in ländlichen Regionen [73]). Diese vielversprechenden Ansätze [6, 74-77] müssen in ihrer Wirksamkeit jedoch noch umfangreich evaluiert werden: Welche Versprechen können eingelöst werden; welche Grenzen zeigen sich; an welchen Stellen ist das Potenzial noch ausbaufähig? Verschiedene, kombinierbare, mitunter überlappende Bereiche lassen sich unterscheiden [70, 78].

\section{Migräne-Apps}

In der heutigen Zeit sind Smartphone-Apps allgegenwärtig. Im Jahr 2018 seien von etwa 50 \% aller Smartphone- 
und Tablet-Nutzer mHealth-Apps heruntergeladen worden [79]. Es gibt eine Reihe von Apps, die explizit für die Edukation und Therapiebegleitung von Kopfschmerzpatienten entwickelt wurden (z. B. digitale Kopfschmerzkalender, Entspannungsübungen, Informationen über die Erkrankung). Eine sorgfältige, an Patientenbedürfnissen ausgerichtete Dokumentation der Attackenverläufe und der Medikamenteneinnahme ist möglich, wodurch eventuell die Adhärenz für die oft mühselige Tagebuchführung erhöht werden kann [80]. Ob dies tatsächlich der Fall ist, bleibt offen [81]. Berichtet wird eine höhere Akzeptanz bei den Benutzern, eine sorgfältigere Dokumentation und größere Effizienz bei der elektronischen Tagebuchführung [80, 82-85]. Symptome lassen sich nach den Kriterien der Klassifikation der IHS [86] erheben und bieten damit eine Unterstützung bei der diagnostischen Einordnung. Die Erstellung eines Trainingsplans und einer daran orientierten Erinnerungs- und Rückmeldefunktion könnte die Motivation für die Durchführung von Ausdauersport erhöhen [87]. Interaktive Illustrationen bei physiotherapeutischen Anleitungen könnten bei der selbstständigen Durchführung im heimischen Setting helfen. Die Vermittlung von PMR mittels Audiodateien über ein Smartphone (App „RELAXaHEAD“) wurde kürzlich durch eine New Yorker Forschergruppe untersucht. Für Migränepatienten, die mindestens 2-mal pro Woche PMR-Übungen durchführten, zeigte sich eine durchschnittliche Verminderung der Migränetage um 4 Tage; bei denen, die weniger übten, waren es nur 2 Tage [88]. Neben Entspannungsübungen haben Apps das Potenzial, KVT-Komponenten anzubieten; durch anschließbare Messfühler ist auch die Umsetzung von Biofeedback möglich [70].

Kopfschmerz-Apps sind in wissenschaftlichen Studien bisher selten untersucht worden [89], ebenso mögliche Nebenwirkungen [90]. Es fehlt an wissenschaftlicher Expertise und es bedarf einheitlicher Qualitätsstandards [87, 91]. Eine Übersichtsarbeit zu Kopfschmerztagebuch-Apps bezeichnet die Evidenz bzgl. Wirksamkeit und klinischer Sicherheit als sehr schwach [92]. Die Investition in weitere Forschung scheint allerdings aus verschiedenen Aspekten lohnenswert: schnelle Verfügbarkeit, die Möglichkeit der umfangreichen Datenerhebung zu Forschungszwecken $[77,93]$ sowie gute Akzeptanz und unter bestimmten Voraussetzungen gesteigerte Adhärenz bei den Nutzern [94]. Vorhersagemodelle individueller Kopfschmerzattacken könnten einem präemptivem Ansatz gerecht werden, Attacken schon vor der Entstehung zu unterbinden [68]. Erste beispielhafte Ansätze sind in Deutschland Mira (Migräne Radar, https://www.migraene-radar.de/), welches das Melden von Migräneattacken und das Abrufen individueller Statistiken ermöglicht [77], bzw. im Vereinigten Königreich die Studie „Cloudy with a Chance of Pain” [93] zum Zusammenhang von Wetter und chronischem Schmerz (https://www.cloudywithachanceofpain.com/).

\section{Telemedizin}

In Gebieten mit geringer Therapeutendichte bzw. Besiedelung, die persönliche Konsultierungen erschweren bzw. kostenintensiv machen, eröffnet Telemedizin neue Möglichkeiten (z. B. durch Kommunikation per Video, Telefon, Textnachrichten oder E-Mail). Eine norwegische Studie konnte für telemedizinische Arztberatung im Vergleich zur Standardtherapie eine Reduktion des finanziellen und zeitlichen Aufwands und eine hohe Akzeptanz des Verfahrens für Kopfschmerzpatienten demonstrieren [73]. In einer Folgeuntersuchung erwies sich die telemedizinische Arztberatung bei nicht akuten Kopfschmerzen bzgl. Effizienz und Sicherheit im Vergleich zum klassischen Arztbesuch als gleichwertig. Schätzungsweise würde in nur einem von 20200 Fällen telemedizinischer Kontakte ein sekundärer Kopfschmerz übersehen werden [95].

\section{Internetbasierte Therapie}

Internetbasierte Therapien für Kopfschmerzbetroffene werden in der Regel von einem Therapeuten via Telefon oder E-Mail angeleitet. Der zeitliche Rahmen und der inhaltliche Aufbau der Programme können sehr unterschiedlich sein; häufig kommen Beratung und Führung zum Einsatz. Ein Review von 2016 untersuchte den Einsatz von elektronisch vermittelten VT-Interventionen bei primären Kopfschmerzerkrankungen. Es kamen überwiegend KVT-Formate zum Einsatz, zumeist über das Internet vermittelt. Trotz guter Akzeptanz und Durchführbarkeit, sind Aussagen zu Wirksamkeit und Adhärenz jedoch begrenzt. Weiterhin war es nicht möglich, zu bewerten, inwiefern die Nutzung dieser digitalen Angebote mit einer regulären Behandlung durch einen Therapeuten vergleichbar ist [89]. Stabile Therapieeffekte im Follow-up und eine signifikante Triptaneinnahmereduktion konnte jedoch eine Untersuchung aus jüngerer Zeit demonstrieren [96]. Zusammenfassend zeigen Studien eine Reduktion von Kopfschmerzfrequenz und -intensität, jedoch auch eine hohe Abbruchrate und keinen Effekt auf depressives oder ängstliches Erleben bei Personen mit chronischem Kopfschmerz. Klare Schlussfolgerungen sind aufgrund fehlender, groß angelegter Studien nicht möglich [89]. Zu ähnlichen Ergebnissen kam ein aktualisierter Cochrane-Review über die Wirksamkeit digital vermittelter psychologischer Therapien bei Kindern und Jugendlichen mit Kopfschmerzen und anderen Schmerzdiagnosen [97].

\section{Informationsangebote im Internet}

Informative Inhalte zum Thema Kopfschmerz finden sich auf vielen Seiten im Internet. Betroffene informieren sich eigeninitiativ und nutzen Expertenforen, um offene Fragen zu klären [98]. Statt von einer eigenen psychologischen Therapieform zu sprechen, sollte man Informationsangebote im Internet als niederschwelliges therapeutisches Angebot verstehen, welches psychoedukative Elemente mit anwendungsbasierten, verhaltenstherapeutischen Komponenten vereint [78]. Internetseiten sollten immer evidenzbasiert sein. Nicht zu unterschätzen ist zudem der Ein- 
fluss dieser auf therapeutisch relevante Patientenvariablen wie Selbstwirksamkeitserwartung, Ursachenattribuierung und Optimismus [55].

Die Ausweitung der psychologischen Kopfschmerztherapie und Therapiebegleitung auf weitere digitale Technologien findet konkrete Anwendung. So beschäftigt sich eine Forschungsgruppe mit einer Sensortechnologie, welche zur Vorhersage von Migräneattacken entwickelt wurde [99]. Auch ist der Einsatz virtueller Realitäten in der Behandlung von Schmerzen und Kopfschmerzen möglich, mit Potenzial in der Zukunft [100-102]. Es bleibt festzuhalten, dass mit eHealth und mHealth vielversprechende Möglichkeiten bei der verhaltenstherapeutischen Behandlung von Migräne und Kopfschmerzen zur Verfügung stehen. Die Durchführbarkeit und Akzeptanz dieser Verfahren erscheinen ausreichend gegeben. Weitere Studie müssen jedoch durchgeführt werden, um verlässliche Wirksamkeitsnachweise zu erbringen. Besonders im Bereich der Smartphone-Apps gilt es die Lücke zwischen kommerziell verfügbaren und wissenschaftlich entwickelten und validierten Apps zu schließen [71]. Wesentliche Aspekte bei der Konzipierung sollten hierbei angemessene Benutzerfreundlichkeit, die Wahrung des Datenschutzes, ein auf die spezielle Benutzergruppe ausgerichtetes, funktionelles Design und die enge Zusammenarbeit mit ärztlichen, psychologischen und technischen Experten sein [71, 91, 92].

\section{FAZIT}

Die wissenschaftlichen psychologischen Behandlungsmethoden bei Kopfschmerz sind eng mit der Entwicklung der Psychotherapie verbunden und haben ursprünglich vor allem unspezifische Wirkfaktoren genutzt. Mit der Entwicklung einer Schmerzpsychotherapie und der damit verbundenen Herausbildung spezifischer Methoden konnten allgemeine Verfahren durch spezifische(re) ersetzt bzw. ergänzt werden. So können beim Bio-/Neurofeedback neben der allgemeinen Entspannungswirkung auch kopfschmerzspezifische Parameter (z. B. Gefäßweite, CNV) verwendet werden. Auch die Etablierung kopfschmerzspezifischer KVT-Programme (z.B. Triggermanagement) leistete hier einen Beitrag. Zukunftsperspektiven sind weitere Ausdifferenzierungen sowie eine maßgeschneiderte Psychotherapie, bei der jeder Patient ein individuelles Therapieprogramm erhält, idealerweise in interdisziplinärer Zusammenarbeit. Eine Einheitstherapie für alle Patienten ist nicht zielführend. Aktuelletechnologische Entwicklungen werden vermutlich die Therapie in Zukunft verändern, wobei die Studienlage zurzeit kaum eindeutige Schlussfolgerungen zulässt und noch einige Herausforderungen (z. B. Qualitätssicherung, Adhärenz) gemeistert werden müssen. Neuere Arbeiten zur Evidenzlage psychologischer Verfahren in der Kopfschmerzbehandlung können als Grundlage einer Diskussion zur Optimierung von Wirkungsforschung angesehen werden. Zukünftige Studien sollten relevante uni- und multimodale Wirkfaktoren berücksichtigen.

\section{Wissenschaftlich verantwortlicher Autor}

Wissenschaftlich verantwortlich gemäß Zertifizierungsbedingungen für diesen Beitrag ist Dr. med. Torsten Kraya, Leipzig.

\section{Interessenkonflikt}

\author{
Erklärung zu finanziellen Interessen des Erstautors \\ Forschungsförderung erhalten: nein. Honorare/geldwerten \\ Vorteil für Referententätigkeit erhalten: ja; Bezahlter \\ Berater/interner Schlungsreferent/Gehaltsempfänger: ja; \\ Patent/Geschäftsanteile/Aktien (Autor/Partner, Ehepartner, \\ Kinder) an Firma: nein \\ Erklärung zu nicht finanziellen Interessen des Erstautors \\ Organisation: Universitätsklinik für Psychiatrie und \\ Psychotherapie, Universität Tübingen (LEAD Graduate \\ School \& Research Network), Position: Postdoktorand, \\ Nachwuchsgruppenleiter; Mitgliedschaften: DMKG e.V. (hier \\ auch kooptiertes Mitglied im Präsidium), LEAD Graduate \\ School \& Research Network (hier auch Mitglied in Vorstand), \\ DGPA e.V., Society for fNIRS, DGPs e.V. \\ Erklärung zu finanziellen Interessen von Dr. med. Torsten \\ Kraya: Forschungsförderung erhalten: nein. Honorare/ \\ geldwerten Vorteil für Referententätigkeit erhalten: ja; \\ Bezahlter Berater/interner Schlungsreferent/Gehaltsempfän- \\ ger: ja; Patent/Geschäftsanteile/Aktien (Autor/Partner, \\ Ehepartner, Kinder) an Firma: nein \\ Erklärung zu nicht finanziellen Interessen von Dr. med. \\ Torsten Kraya: Der Autor gibt an, dass kein Interessenkonflikt \\ besteht.
}

\section{Korrespondenzadresse}

\author{
Dr. phil. Thomas Dresler \\ Klinik für Psychiatrie und Psychotherapie \\ Universität Tübingen \\ Calwerstr. 14, 72076 Tübingen \\ thomas.dresler@med.uni-tuebingen.de
}

\section{Literatur}

[1] Kropp P, Meyer B, Dresler T, et al. [Relaxation techniques and behavioural therapy for the treatment of migraine : Guidelines from the German Migraine and Headache Society]. Schmerz 2017; 31(5): 433-47 
[2] Diener H-C, Gaul C, Kropp P, et al. Therapie der Migräneattacke und Prophylaxe der Migräne, S1-Leitlinie, 2018 [Therapy of migraine attacks and migraine prophylaxis]. In: DGN (Hrsg.) Leitlinien für Diagnostik und Therapie in der Neurologie 2018

[3] Straube A, Brössner G, Diener H-C, et al. Therapie des episodischen und chronischen Kopfschmerzes vom Spannungstyp und anderer chronischer täglicher Kopfschmerzen, S1-Leitlinie, 2015 [Treatment of episodic and chronic tension-type headache and other chronic daily headaches]. In: DGN (Hrsg.) Leitlinien für Diagnostik und Therapie in der Neurologie 2015

[4] May A, Brössner G, Diener H-C, et al. Clusterkopfschmerz und trigeminoautonome Kopfschmerzen, S1-Leitlinie, 2015 [Cluster headache and trigeminal autonomic headache]. In: DGN (Hrsg.) Leitlinien für Diagnostik und Therapie in der Neurologie 2015

[5] Diener H-C, Gaul C, Kropp P, et al. Kopfschmerz bei Übergebrauch von Schmerz- oder Migränemitteln (Medication Overuse Headache $=\mathrm{MOH}$ ), S1-Leitlinie, 2018 [Headache due to overuse of analgesics and anti-migraine agents]. In: DGN (Hrsg.) Leitlinien für Diagnostik und Therapie in der Neurologie 2018

[6] Penzien DB, Irby MB, Smitherman TA, et al. Well-Established and Empirically Supported Behavioral Treatments for Migraine. Curr Pain Headache Rep 2015; 19(7): 34

[7] Sullivan A, Cousins S, Ridsdale L. Psychological interventions for migraine: a systematic review. J Neurol 2016; 263(12): 2369-77

[8] Lee HJ, Lee JH, Cho EY, et al. Efficacy of psychological treatment for headache disorder: a systematic review and meta-analysis. J Headache Pain 2019; 20(1): 17

[9] Sharpe L, Dudeney J, Williams ACC, et al. Psychological therapies for the prevention of migraine in adults. Cochrane Database Syst Rev 2019; 7: CD012295

[10] Fisher E, Law E, Dudeney J, et al. Psychological therapies for the management of chronic and recurrent pain in children and adolescents. Cochrane Database Syst Rev 2018; 9 : CD003968

[11] Nestoriuc Y, Rief W, Martin A. Meta-analysis of biofeedback for tension-type headache: efficacy, specificity, and treatment moderators. J Consult Clin Psychol 2008; 76(3): 379-96

[12] Evers S. Die Medizingeschichte der Migräne. migräne magazin 2011; 52

[13] Koehler PJ, Boes CJ. A history of non-drug treatment in headache, particularly migraine. Brain 2010; 133(Pt 8): 2489-500

[14] Rapoport A, Edmeads J. Migraine: the evolution of our knowledge. Arch Neurol 2000; 57(8): 1221-3

[15] Villalon CM, Centurion D, Valdivia LF, et al. An introduction to migraine: from ancient treatment to functional pharmacology and antimigraine therapy. Proc West Pharmacol Soc 2002; 45: 199-210

[16] Stumm G. Geschichte, Paradigmen und Methoden der Psychotherapie. In: Slunecko T (Hrsg.) Psychotherapie. Eine Einführung. Wien: facultas.wuv Universitätsverlag 2017; 33-90

[17] Margraf J. Grundlagen - Hintergründe und Entwicklung. In: Margraf J, Schneider S (Hrsg.) Lehrbuch der Verhaltenstherapie, Band 1 (4 Aufl). Berlin: Springer; 2018, 13-35

[18] Gatchel RJ, Peng YB, Peters ML, et al. The biopsychosocial approach to chronic pain: scientific advances and future directions. Psychol Bull 2007; 133(4): 581-624
[19] Kropp P, Dresler T, Niederberger U. Psychosomatik der Kopfschmerzen. Psychotherapeut 2014; 59(1): 57-71

[20] Kropp P, Niederberger U. Schmerz. In: Brähler E, Strauß B (Hrsg.) Grundlagen der Medizinischen Psychologie - Medizinische Psychologie. Göttingen: Hogrefe; 2012

[21] Meyer B, Keller A, Wohlbier HG, et al. Progressive muscle relaxation reduces migraine frequency and normalizes amplitudes of contingent negative variation (CNV). J Headache Pain 2016; 17: 37

[22] Andrasik F. What does the evidence show? Efficacy of behavioural treatments for recurrent headaches in adults. Neurol Sci 2007; 28 Suppl 2: S70-7

[23] Fritsche G, Kroner-Herwig B, Kropp P, et al. [Psychological therapy of migraine: systematic review]. Schmerz 2013; 27(3): 263-74

[24] Klan T, Liesering-Latta E, Gaul C, et al. An Integrative Cognitive Behavioral Therapy Program for Adults With Migraine: A Feasibility Study. Headache 2019; 59(5): 741-55

[25] Andrasik F. The essence of biofeedback, relaxation and hypnosis - Psychosocial Aspects of Pain: A Handbook for Healthcare providers. Progress in Pain Research and Management. Seattle: IASP Press; 2004

[26] Tracey I, Ploghaus A, Gati JS, et al. Imaging attentional modulation of pain in the periaqueductal gray in humans. J Neurosci 2002; 22(7): 2748-52

[27] Borkovec TD, Costello E. Efficacy of applied relaxation and cognitive-behavioral therapy in the treatment of generalized anxiety disorder. J Consult Clin Psychol 1993; 61(4): 611-9

[28] Krampen G. Einführungskurse zum Autogenen Training: Ein Lehr- und Übungsbuch für die psychosoziale Praxis. Göttingen: Hogrefe; 1998

[29] Woelm E. Anleitung zum Autogenen Training. In: Hypnos - Institut für Hypnosys-temische Lösungen. 2011; 11.

[30] Stetter F, Kupper $S$. Autogenic training: a meta-analysis of clinical outcome studies. Appl Psychophysiol Biofeedback 2002; 27(1): 45-98

[31] Seo E, Hong E, Choi J, et al. Effectiveness of autogenic training on headache: A systematic review. Complement Ther Med 2018; 39: 62-7

[32] Kang E-H, Ahn J-Y, Koo M-S, et al. Biofeedback-assisted autogenic training for chronic tension-type headache in a Korean population. Journal of Korean Neuropsychiatric Association. 2008; 47(3): 247-53

[33] Kang EH, Park JE, Chung CS, et al. Effect of biofeedback-assisted autogenic training on headache activity and mood states in Korean female migraine patients. J Korean Med Sci 2009; 24(5): 936-40

[34] Pickering G, Creac'h C, Radat F, et al. Autogenic training in patients treated for chronic headache: A randomised clinical trial. Journal of Pain Management 2012; 5(2): 195-205

[35] Bernstein DA, Borkovec TD. Progressive relaxation training: A manual for the helping professions. Champaign, IL, US: Research Press; 1973

[36] Diezemann A. Entspannungsverfahren bei chronischem Schmerz [Relaxation techniques for chronic pain]. Schmerz 2011; 25(4): 445-53

[37] Maercker A, Krampen G. Entspannungsverfahren. In: Margraf J, Schneider S (Hrsg.) Lehrbuch der Verhaltenstherapie, Band 1 (4 Aufl). Berlin: Springer; 2018, 395-401

[38] Stück M, Rigotti T, Mohr G. Untersuchungen der Wirksamkeit eines Belastungsbewältigungstrainings für den 
Lehrberuf. Psychologie in Erziehung und Unterricht. 2004; 51: 236-45

[39] Grawe K, Donati R, Bernauer F. Psychotherapie im Wandel. Von der Konfession zur Profession. Göttingen Hogrefe; 1994

[40] Benson H. The relaxtion response. New York: Avon; 1975

[41] Meyer B, Niederberger U, Sorgenfrei V, et al. Kopfschmerz unter (Selbst-)kontrolle [Headache and self-regulation]. MMW Fortschr Med 2017; 159(2): 62-5

[42] Kropp P, Gerber WD, Keinath-Specht A, et al. Behavioral treatment in migraine. Cognitive-behavioral therapy and blood-volume-pulse biofeedback: a cross-over study with a two-year follow-up. Funct Neurol 1997; 12(1): 17-24

[43] Kropp P, Niederberger U. Theoretische Konzepte und Wirkmechanismen. In: Martin A, Rief W (Hrsg.) Wie wirksam ist Biofeedback? Bern: Huber; 2008, 43-9

[44] Wilken B. Methoden der kognitiven Umstrukturierung: Ein Leitfaden für die psychotherapeutische Praxis. Stuttgart: Kohlhammer Verlag; 2018

[45] Basler HD. Psychologische Therapie bei Kopf-und Rückenschmerzen: Das Marburger Schmerzbewältigungsprogramm zur Gruppen-und Einzeltherapie. Köln: Quintessenz Verlag; 1998

[46] Denecke H, Kröner-Herwig B. Kopfschmerz-Therapie mit Kindern und Jugendlichen: Ein Trainingsprogramm: Göttingen: Hogrefe; 2000

[47] Richter M, Gruhl E, Lautenschlager E, et al. [DreKiP - an outpatient treatment program for children and adolescents with headache]. Schmerz 2018; 32(1): 17-29

[48] Pellegrino ABW, Davis-Martin RE, et al. Perceived triggers of primary headache disorders: A meta-analysis. Cephalalgia 2018; 38(6): 1188-98

[49] Martin PR. Behavioral management of migraine headache triggers: learning to cope with triggers. Curr Pain Headache Rep 2010; 14(3): 221-7

[50] Martin PR, MacLeod C. Behavioral management of headache triggers: Avoidance of triggers is an inadequate strategy. Clin Psychol Rev 2009; 29(6): 483-95

[51] Martin PR. How do trigger factors acquire the capacity to precipitate headaches? Behav Res Ther 2001; 39(5): 545-54

[52] Martin PR, Lae L, Reece J. Stress as a trigger for headaches: relationship between exposure and sensitivity. Anxiety Stress Coping 2007; 20(4): 393-407

[53] Martin PR, Reece J, Forsyth M. Noise as a trigger for headaches: relationship between exposure and sensitivity. Headache 2006; 46(6): 962-72

[54] Martin PR, Reece J, Callan M, et al. Behavioral management of the triggers of recurrent headache: a randomized controlled trial. Behav Res Ther 2014; 61: 1-11

[55] Martin PR, Timmings HY. Effect of Headache Websites on Locus of Control and Self-efficacy of Readers. Aust Psychol 2017; 52(1): 72-80

[56] Klan T, Liesering-Latta E. Kognitiv-verhaltenstherapeutisches Migränemanagement (MIMA). Ein Behandlungsmanual zur Krankheitsbewältigung und Attackenprophylaxe bei Migräne. Göttingen: Hogrefe; 2019

[57] Hildebrandt ], Pfingsten M, Franz C, et al. [Multidisciplinary treatment program for chronic low back pain, part 1. Overview]. Schmerz 1996; 10(4): 190-203

[58] Arnold B, Brinkschmidt T, Casser HR, et al. [Multimodal pain therapy: principles and indications]. Schmerz 2009; 23(2): $112-20$

[59] Scascighini L, Toma V, Dober-Spielmann S, et al. Multidisciplinary treatment for chronic pain: a systematic review of interventions and outcomes. Rheumatology (Oxford) 2008; 47(5): 670-8

[60] Gaul C, Liesering-Latta E, Schafer B, et al. Integrated multidisciplinary care of headache disorders: A narrative review. Cephalalgia 2016; 36(12): 1181-91

[61] Fritsche G, Gaul C. Multimodale Schmerztherapie bei chronischen Kopfschmerzen: Interdisziplinäre Behandlungskonzepte. Stuttgart: Georg Thieme Verlag; 2013

[62] Gaul C, Visscher CM, Bhola R, et al. Team players against headache: multidisciplinary treatment of primary headaches and medication overuse headache. J Headache Pain 2011; 12(5): 511-9

[63] Kindelan-Calvo P, Gil-Martinez A, Paris-Alemany A, et al. Effectiveness of therapeutic patient education for adults with migraine. A systematic review and meta-analysis of randomized controlled trials. Pain Med 2014; 15(9): 1619-36

[64] Wallasch TM, Kropp P. Multidisciplinary integrated headache care: a prospective 12-month follow-up observational study. J Headache Pain 2012; 13(7): 521-9

[65] Caroli A, Klan T, Gaul C, et al. Die Erfassung von Triggerempfindlichkeit und -vermeidung bei primären Kopfschmerzen: Entwicklung und psychometrische Erprobung einer deutschen Version des Headache Triggers Sensitivity and Avoidance Questionnaire (HTSAQ-G). Verhaltenstherapie 2019; Epub ahead

[66] Graef JE, Rief W, French DJ, Nilges P, Nestoriuc Y. German Language Adaptation of the Headache Management Self-Efficacy Scale (HMSE-G) and Development of a New Short Form (HMSE-G-SF). Headache 2015; 55(7): 958-72

[67] Klan T, Bräscher A, Vales A, et al. Measuring strain, disability and coping in Cluster Headache - construction and psychometric testing of a self-assessment questionnaire. in Vorbereitung.

[68] Houle TT, Turner DP, Golding AN, et al. Forecasting Individual Headache Attacks Using Perceived Stress: Development of a Multivariable Prediction Model for Persons With Episodic Migraine. Headache 2017; 57(7): 1041-50

[69] Meißner A. Wie Apps und Algorithmen zunehmend die Medizin bestimmen. NeuroTransmitter 2018; 29: 16-21

[70] Dresler T, Klan T, Kraya T, et al. Aktuelle Entwicklungen in der psychologischen Kopfschmerztherapie. NeuroTransmitter 2018; 29: 58-66

[71] Stubberud A, Linde M. Digital Technology and Mobile Health in Behavioral Migraine Therapy: a Narrative Review. Curr Pain Headache Rep 2018; 22(10): 66

[72] Neeb L, Dresler T. Sollten Patienten Kopfschmerz-Apps verwenden? Schmerzmedizin 2019; 35(4): 10-1

[73] Muller KI, Alstadhaug KB, Bekkelund SI. Acceptability, Feasibility, and Cost of Telemedicine for Nonacute Headaches: A Randomized Study Comparing Video and Traditional Consultations. J Med Internet Res 2016; 18(5): e140

[74] Zhu Q, Liu C, Holroyd KA (eds.) From a traditional behavioral management program to an m-health app: Lessons learned in developing $\mathrm{m}$-health apps for existing health care programs. 2012 4th International Workshop on Software Engineering in Health Care (SEHC); 2012 4-5 June 2012

[75] Luxton DD, McCann RA, Bush NE, et al. mHealth for mental health: Integrating smartphone technology in behavioral healthcare. US: American Psychological Association; 2011, 505-12

[76] Andrasik F. Behavioral treatment of headaches: extending the reach. Neurol Sci 2012;33 Suppl 1: S127-30 
[77] Drescher J, Wogenstein F, Gaul C, et al. Distribution of migraine attacks over the days of the week: Preliminary results from a web-based questionnaire. Acta Neurol Scand 2019; 139(4): 340-5

[78] Kropp P, Meyer B, Meyer W, et al. An update on behavioral treatments in migraine - current knowledge and future options. Expert Rev Neurother 2017; 17(11): 1059-68

[79] Mobile Medical Applications [Internet]. 2018. Available from: https://www.fda.gov/medical-devices/digital-health/ mobile-medical-applications

[80] Stone AA, Shiffman S, Schwartz JE, et al. Patient compliance with paper and electronic diaries. Control Clin Trials 2003; 24(2): 182-99

[81] Seng EK, Prieto P, Boucher G, et al. Anxiety, Incentives, and Adherence to Self-Monitoring on a Mobile Health Platform: A Naturalistic Longitudinal Cohort Study in People With Headache. Headache 2018; 58(10): 1541-55

[82] Burton C, Weller D, Sharpe M. Are electronic diaries useful for symptoms research? A systematic review. J Psychosom Res 2007; 62(5): 553-61

[83] Affleck G, Zautra A, Tennen H, et al. Multilevel daily process designs for consulting and clinical psychology: a preface for the perplexed. J Consult Clin Psychol 1999; 67(5): 746-54

[84] Sorbi MJ, Mak SB, Houtveen JH, et al. Mobile Web-based monitoring and coaching: feasibility in chronic migraine. J Med Internet Res 2007; 9(5): e38

[85] Lewandowski AS, Palermo TM, et al. Comparing diary and retrospective reports of pain and activity restriction in children and adolescents with chronic pain conditions. Clin J Pain 2009; 25(4): 299-306

[86] Headache Classification Committee of the International Headache S. The International Classification of Headache Disorders, 3 rd edition (beta version). Cephalalgia 2013; 33(9): 629-808

[87] Dahlem MA, Roesch A, Neeb L, et al. [What do migraine apps offer?]. MMW Fortschr Med 2018; 160(2): 51-4

[88] Minen MT, Adhikari S, Seng EK, et al. Smartphone-based migraine behavioral therapy: a single-arm study with assessment of mental health predictors. NPJ Digit Med 2019; 2: 46

[89] Minen MT, Torous J, Raynowska J, et al. Electronic behaviora interventions for headache: a systematic review. J Headache Pain 2016; 17: 51

[90] Stevens WJM, van der Sande R, Beijer LJ, et al. eHealth Apps Replacing or Complementing Health Care Contacts: Scoping Review on Adverse Effects. J Med Internet Res 2019; 21(3): e10736

[91] Hundert AS, Huguet A, McGrath PJ, et al. Commercially available mobile phone headache diary apps: a systematic review. JMIR Mhealth Uhealth 2014; 2(3): e36

[92] Mosadeghi-Nik M, Askari MS, Fatehi F. Mobile health (mHealth) for headache disorders: A review of the evidence base. J Telemed Telecare 2016; 22(8): 472-7

[93] Druce KL, McBeth J, van der Veer SN, et al. Recruitment and Ongoing Engagement in a UK Smartphone Study Examining the Association Between Weather and Pain: Cohort Study. JMIR Mhealth Uhealth 2017; 5(11): e168
[94] Ebner H, Schreier G. Medication Adherence and Monitoring. In: Stegemann S, editor. Developing Drug Products in an Aging Society From Concept to Prescribing. Berlin: Springer; 2016; 659-74

[95] Muller KI, Alstadhaug KB, Bekkelund SI. A randomized trial of telemedicine efficacy and safety for nonacute headaches. Neurology 2017; 89(2): 153-62

[96] Sorbi M], Balk Y, Kleiboer AM, et al. Follow-up over 20 months confirms gains of online behavioural training in frequent episodic migraine. Cephalalgia 2017; 37(3): 236-50

[97] Fisher E, Law E, Dudeney J, et al. Psychological therapies (remotely delivered) for the management of chronic and recurrent pain in children and adolescents. Cochrane Database Syst Rev 2019; 4: CD011118

[98] Scherer M, Zitterbart S, Mildenstein K, et al. [What questions do headache patients pose in the internet? Content analysis of an internet expert forum]. Gesundheitswesen 2010; 72(5): e28-32

[99] Pagán J, Zapater M, Ayala JL. Power transmission and workload balancing policies in eHealth mobile cloud computing scenarios. Future Generation Computer Systems 2018; 78: 587-601

[100] Shiri S, Feintuch U, Weiss N, et al. A virtual reality system combined with biofeedback for treating pediatric chronic headache - a pilot study. Pain Med 2013; 14(5): 621-7

[101] Riva G. Virtual environments in clinical psychology. Psychotherapy Theory Research Practice Training 2013; 40(1-2): 68-76

[102] Lange B, Williams M, Fulton I. Virtual reality distraction during pediatric medical procedures. Pediatr Pain Lett 2006; 8(1): 6-10

[103] Kosinski M, Bayliss MS, Bjorner JB, et al. A six-item shortform survey for measuring headache impact: The HIT-6 ${ }^{\mathrm{TM}}$. Quality of Life Research 2003; 12: 963-74

[104] Gandek B, Alacoque J, Uzun V, et al. Translating the ShortForm Headache Impact Test (HIT-6) in 27 countries: Methodological and conceptual issues. Quality of Life Research 2003; 12: 975-9

[105] Bauer B, Evers S, Gralow I, et al. Psychosoziale Beeinträchtigung durch chronische Kopfschmerzen. Nervenarzt 1999; 70: 522-9

[106] Agosti R, Chrubasik JE, Kohlmann T. Der MIDAS-Fragebogen. Ars Medici 2008; 16: 700-1

Bibliografie

DOI https://doi.org/10.1055/a-0989-6551

Nervenheilkunde 2019; 38: 745-756

(c) Georg Thieme Verlag KG Stuttgart · New York

ISSN 0722-1541 


\section{Punkte sammeln auf CM/F.thieme.de}

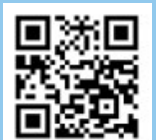

Diese Fortbildungseinheit ist in der Regel 12 Monate online für die Teilnahme verfügbar. Den genauen Einsendeschluss finden Sie unter https://eref.thieme.de/CXDNU35.

Sollten Sie Fragen zur Online-Teilnahme haben, finden Sie unter https://cme.thieme.de/hilfe eine ausführliche Anleitung. Wir wünschen viel Erfolg beim Beantworten der Fragen!

Unter https://eref.thieme.de/CXDNU35 oder über den QR-Code kommen Sie direkt zur Startseite des Wissenstests.

VNR 2760512019156645390

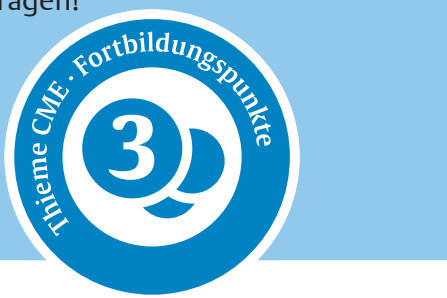

\section{Frage 1}

Welche Aussage zum Autogenen Training ist korrekt? Das Autogene Training ist ...

A eine Methode, um dem Patienten körperliche Prozesse über ein gut wahrnehmbares, meist visuelles oder akustisches Signal zurückzumelden.

B eine Methode, um die Flexibilisierung im Umgang mit potenziellen Triggern zu fördern.

C eine Entspannungstechnik, bei der einzelnen Muskelgruppen für wenige Sekunden angespannt und im Folgenden entspannt werden.

D eine Form der vereinfachten und standardisierten Selbsthypnose.

E ein Entspannungsverfahren, in dem eine Reihe geistiger und körperlicher Übungen (Asanas) bzw. Praktiken wie Meditation und Atemübungen (Pranayama) eingesetzt werden.

\section{Frage 2}

In welcher Entwicklungsphase der Verhaltenstherapie nehmen Meditationstechniken eine zunehmend größere Bedeutung für die Therapie ein?

A Erste Welle der Verhaltenstherapie.

B Zweite Welle der Verhaltenstherapie.

C Dritte Welle der Verhaltenstherapie.

D Vierte Welle der Verhaltenstherapie.

E Fünfte Welle der Verhaltenstherapie.

\section{Frage 3}

Wie ist die Evidenzlage von Psychotherapie bei Kopfschmerzen zu beurteilen?

A Psychodynamische Therapieverfahren sind verhaltenstherapeutischen Verfahren hinsichtlich ihrer Wirksamkeit in der Kopfschmerzbehandlung deutlich überlegen.

B Es gibt Hinweise auf gute Effekte von verhaltenstherapeutischen Verfahren bei primären Kopfschmerzen, wobei die Evidenzlage insgesamt noch nicht zufriedenstellend geklärt ist.

C Psychotherapie ist nur bei sekundären Kopfschmerzen wirksam.

D Alle psychotherapeutischen Verfahren außer Biofeedback sind bei primären Kopfschmerzen sehr gut wirksam und zeichnen sich durch sehr hohe Effektstärken aus.

E Die Evidenz von Psychotherapie in der Behandlung von Kopfschmerzen ist gesichert.

\section{Frage 4}

Was besagt das Trigger Avoidance Model of Headaches (Triggervermeidungsmodell)?

A Das Vermeiden von Triggern verhindert unnötige Belastungen und ist daher der Königsweg in der verhaltenstherapeutischen Behandlung von Kopfschmerzen.

B Das Trigger Avoidance Model of Headaches beschreibt, wie ein Übermaß an Triggerbelastungen zur Entstehung einer Clusterkopfschmerzattacke führt.

C Eine übermäßige Vermeidung von Kopfschmerzauslösern kann zu einer Sensitivierung hinsichtlich dieser Auslöser führen und somit zur Chronifizierung einer primären Kopfschmerzerkrankung beitragen.

D Bei Kopfschmerzen sollte körperliche Aktivität vermieden werden, da dies zu einer erhöhten kardiovaskulären Aktivierung und Verstärkung der Kopfschmerzen führt.

E Das Trigger Avoidance Model of Headaches erklärt, wie muskuläre Triggerpunkte zu Kopfschmerz vom Spannungstyp führen. 


\section{Punkte sammeln auf CME.thieme.de}

Fortsetzung $\ldots$

\section{Frage 5}

Welche Aussage zur psychologischen Kopfschmerzdiagnostik ist richtig?

A Die psychologische Kopfschmerzdiagnostik umfasst verschiedene Bereiche, darunter die kopfschmerzassoziierte Beeinträchtigung und die Krankheitsverarbeitung.

B Eine gute psychologische Kopfschmerzdiagnostik kann bildgebende Verfahren ersetzen.

C Psychologische Kopfschmerzdiagnostik führt zur unnötigen Stigmatisierung von Patienten und sollte daher vermieden werden.

D Die Anwendung eines Fragebogens reicht in der Regel aus, da psychologische Faktoren meistens eindimensional sind.

E Für jede Kopfschmerzerkrankung gibt es ein spezifisches Testverfahren.

\section{Frage 6}

Was trifft für die Kognitive Verhaltenstherapie im Kontext der Behandlung von Kopfschmerzen zu?

A Die Entwicklung der kognitiven Verhaltenstherapie ist mit der sogenannten „zweiten Welle“ stehen geblieben.

B Das Triggermanagement ist der zentrale Bestandteil einer jeden Kognitiven Verhaltenstherapie.

C Kognitive Verhaltenstherapie und Entspannungsverfahren können nicht parallel angewendet werden.

D Es werden zunehmend spezifische Behandlungsprogramme entwickelt.

E Kognitive Verhaltenstherapie sollte nur dann angewendet werden, wenn der Patient auf eine medikamentöse Behandlung nicht anspricht.

\section{Frage 7}

Welchen Stellenwert haben psychologische Behandlungsverfahren in den vorhandenen Leitlinien zur Kopfschmerzbehandlung?

A Nicht in allen kopfschmerzrelevanten Leitlinien werden psychologische Verfahren als indiziert genannt.

B Psychologische Behandlungsverfahren sind in allen kopfschmerzrelevanten Leitlinien als wichtiger Therapieansatz verortet.

C Bislang werden psychotherapeutische Verfahren in keiner kopfschmerzspezifischen Leitlinie erwähnt.

D Psychotherapie ist das zentrale Behandlungselement in fast allen Leitlinien zur Kopfschmerzbehandlung.

E Nur in der S1-Leitlinie zur Behandlung von Clusterkopfschmerz und trigeminoautonomen Kopfschmerzen werden psychologische Behandlungselemente aufgeführt.

\section{Frage 8}

In welchen Fällen bietet sich eHealth/mHealth bei Kopfschmerzen besonders an?

A Wenn ein Äquivalent zur speziellen Schmerzpsychotherapie notwendig ist, sofern diese sonst schwierig zu initiieren ist.

B Für das Training technischer Fertigkeiten bei Menschen ohne Smartphone.

C Solche Anwendungen sind ausschließlich bei chronifizierten Kopfschmerzen sinnvoll.

D Zur Vermittlung nicht medikamentöser Konzepte mit dem Potenzial einer insgesamt verbesserten Patientenversorgung.

E Wenn der Patient einen Kopfschmerz durch Medikamentenübergebrauch entwickelt.

\section{Frage 9}

Was können Migräne-Apps leisten?

A Sie sollen perspektivisch die herkömmliche Standardtherapie ersetzen.

B Sie dienen häufig der Edukation, Therapiebegleitung und Dokumentation von Attacken und Medikamenteneinnahme.

C Sie ermöglichen die Erstellung von Rezepten für die Schmerzmedikation.

D Migräne-Apps haben keinerlei relevante Effekte und die Inhalte sind nicht nützlich.

E Migräne-Apps ermöglichen den Ausschluss eines sekundären Kopfschmerzes.

\section{Frage 10}

Was ist „multimodale“ Kopfschmerztherapie?

A Die Einnahme von Multivitaminpräparaten zur Nahrungsergänzung.

B Die Kombination von Medikamenten und homöopathischen Präparaten.

C Der Einsatz von visuellen, akustischen, taktilen und olfaktorischen Reizen.

D Der Einsatz von ambulanter, teilstationärer und stationärer Therapie.

E Der Einsatz von Verfahren aus verschiedenen Fachrichtungen. 